\title{
Efficacy evaluation of vaginal-assisted laparoscopic radical hysterectomy and laparoscopic radical hysterectomy for cervical cancer: a single-center retrospective case series study
}

\author{
Huimin Wang ${ }^{1 \#}$, Dianzhen Li ${ }^{1 \#}$, Chunyan Wang ${ }^{1}$, Xiaobin Wang ${ }^{1}$, Mingxin Yu ${ }^{1}$, Xin Zhang ${ }^{1}$, Liankun $\mathrm{Li}^{1}$, \\ Qingdong Zeng', Zaiqiu Long ${ }^{1}$, Wei Zheng ${ }^{1}$, Guangcong Liu ${ }^{2}$, Danbo Wang ${ }^{1}$ \\ ${ }^{1}$ Department of Gynecology, Liaoning Cancer Hospital \& Cancer Hospital of China Medical University, Shenyang, China; ${ }^{2}$ Department of \\ Epidemiology, Liaoning Cancer Hospital \& Cancer Hospital of China Medical University, Shenyang, China \\ Contributions: (I) Conception and design: H Wang, D Li, D Wang; (II) Administrative support: D Wang, G Liu; (III) Provision of study materials or \\ patients: D Wang, C Wang, X Wang, M Yu, X Zhang, L Li, Q Zeng, Z Long, W Zheng; (IV) Collection and assembly of data: H Wang, D Li; (V) \\ Data analysis and interpretation: H Wang, D Li, G Liu; (VI) Manuscript writing: All authors; (VII) Final approval of manuscript: All authors. \\ \#These authors contributed equally to this work. \\ Correspondence to: Danbo Wang; Guangcong Liu. Liaoning Cancer Hospital \& Cancer Hospital of China Medical University, No. 44 Xiaoyan Rd, \\ Dadong District, Shenyang 110042, China. Email: wangdanbo@cancerhosp-ln-cmu.com; liuguangcong@cancerhosp-ln-cmu.com.
}

Background: To investigate the survival outcomes of abdominal radical hysterectomy (ARH), laparoscopic radical hysterectomy (LRH), and vaginal-assisted laparoscopic radical hysterectomy (VALRH) in the treatment of cervical cancer patients.

Methods: This was a retrospective study. We collected the clinical data of 654 patients with cervical cancer (406 ARH, $172 \mathrm{LRH}$, and $76 \mathrm{VALRH}$ ), then compared the effects of different surgical methods on recurrence and survival.

Results: Total overall survival (OS) were no significant differences in three groups $(\mathrm{P}>0.05)$. Total diseasefree survival (DFS) was significantly higher in ARH group than in LRH group [hazard ratio (HR) $=2.8,95 \%$ confidence interval (CI): 1.199-3.607, $\mathrm{P}=0.004]$; however, there were no significant differences between the VALRH (94.7\%) and ARH (93.3\%) groups. Subgroup stratification analysis showed that the overall recurrence rate in LRH group was significantly higher than that of the ARH groups for patients with a tumor size from $\geq 2$ to $<4 \mathrm{~cm}$, negative postoperative lymph nodes, and no postoperative adjuvant therapy (all $\mathrm{P}<0.05$ ). However, in the subgroup with tumor sizes of $\geq 2,<4$, and $\geq 4 \mathrm{~cm}$, no matter whether the lymph nodes were positive or not, and those with no postoperative supplementary adjuvant therapy, LRH was associated with a significantly higher local pelvic recurrence rate than ARH (all $\mathrm{P}<0.05$ ). No significant differences between VALRH and $\mathrm{ARH}$ in any of the subgroup analyses (all $\mathrm{P}>0.05$ ). A Cox analysis indicated that LRH increased the risk of overall and local pelvic recurrence after surgery compared with ARH (HR =2.338, 95\% CI: 1.186-4.661, $\mathrm{P}=0.014 ; \mathrm{HR}=10.313$, 95\% CI: 2.839-37.460, $\mathrm{P}<0.001$ ); however, no significant difference between VALRH and ARH (all $\mathrm{P}>0.05$ ). Sensitivity analysis of surgeons did not change the conclusions.

Conclusions: Our analyses showed that the local pelvic recurrence rates and overall recurrence rates of LRH were significantly higher than ARH. VALRH could avoid tumor intraperitoneal exposure and achieve the same tumor prognosis as open surgery. By improving the standardization of minimally invasive surgery for early cervical cancer and paying close attention to the tumor-free concept, minimally invasive radical hysterectomy may achieve the same tumor outcome as open surgery.

Keywords: Vaginal-assisted laparoscopic radical hysterectomy (VALRH); laparoscopic radical hysterectomy (LRH); abdominal radical hysterectomy (ARH); cervical cancer; efficacy

Submitted Nov 09, 2021. Accepted for publication Dec 23, 2021.

doi: $10.21037 /$ atm-21-6450

View this article at: https://dx.doi.org/10.21037/atm-21-6450 


\section{Introduction}

Cervical cancer is a very common condition in gynecological oncology. Radical hysterectomy combined with pelvic lymph node dissection, is one of the main treatment methods for early cervical cancer (1). In addition to the traditional abdominal radical hysterectomy (ARH), several minimally invasive surgical methods have been developed in recent years, including transvaginal radical hysterectomy (VRH), which has gradually been replaced by laparoscopic radical hysterectomy (LRH) due to technical difficulties. LRH was first introduced in the 1980s and was rapidly promoted due to the fact that its long arm and visual amplification were highly advantageous for cervical cancer surgery. Another benefit of LRH is that it allows patients to avoid the huge trauma of surgery. LRH has become very popular in China, as one of the countries with a relatively high incidence of cervical cancer. The widespread application of LRH has led to a multitude of reports relating to technical advantages (2) and efficacy evaluation (3). However, in 2018, the results of the Laparoscopic Approach to Cervical Cancer (LACC) trial reported by Ramirez et al. (4) showed that minimally invasive surgery was associated with higher recurrence and mortality rates, which caused significant concern in the academic community. Around the same time, Melamed et al. (5) published the results of the Real World Study (RWS), which reached the same conclusion as the LACC trial. Therefore, serious concerns were raised over the efficacy of minimally invasive surgery for cervical cancer. However, following the LACC trial, reports demonstrating that there was no difference in survival rates between invasive surgery and open surgery for patients with early cervical cancer were published $(6,7)$. These reports, and the technical advantages of minimally invasive surgery, suggest that minimally invasive techniques could potentially be applied to patients with cervical cancer. Therefore, there is a clear need to investigate whether minimally invasive surgery for cervical cancer is feasible after improving the technical factors.

When analyzing the reasons underlying the poor survival outcomes associated with minimally invasive surgery for cervical cancer, one of the most significant issues is whether this technique can guarantee an intraoperative tumorfree environment. The direct compression of cervical tumors by cup-shaped uterine manipulators $(8,9)$ and vaginal dissection performed in a carbon dioxide $\left(\mathrm{CO}_{2}\right)$ pneumoperitoneum environment to expose tumor cells $(10,11)$, are factors that are believed to be directly related to the poor prognosis of patients undergoing minimally invasive surgery. Therefore, further evidence is needed to ascertain whether relevant corrective measures can enhance minimally invasive surgery for patients with cervical cancer and improve prognosis. VRH is a classical minimally invasive surgical method for patients with cervical cancer and was first reported by Dargent in 1994. This technique has recently attracted greater attention, along with laparoscopic pelvic lymph node resection (12). VRH has the advantage of achieving tumor-free surgery by using a vaginal cuff to close lesions, but is also associated with a long learning curve due to its retrograde anatomical pathway, which has reduced its clinical popularity. Compared with LRH, all operations including vaginal resection are performed under laparoscopy, while VRH is all performed in the vagina. Vaginal-assisted laparoscopic radical hysterectomy (VALRH) refers to lymph node dissection, complete ligamentectomy for radical hysterectomy until vaginal disjunction were performed under laparoscopy, then through the vagina to remove the residual sacral ligament and visico-cervical ligament, and complete radical hysterectomy was subsequently performed, which can not only play the minimally invasive advantages of laparoscopy, but also play the no tumor advantages of vaginal surgery. At the same time, the operation through the vagina is less and the difficulty is lower than VRH. In our center, open surgery, laparoscopic surgery, and vaginal surgery are all being developed in a balanced manner for patients with early-stage cervical cancer. In order to simplify vaginal surgery and facilitate surgery transmission, we have performed VALRH since 2015, which has now become one of our conventional techniques.

At present, there are very few publications relating to this particular technique, The comparative analysis of VALRH, ARH and LRH in the treatment of cervical cancer has not been reported. In this study, we retrospectively collected clinical and follow-up data from patients who underwent radical cervical cancer surgery, including ARH, LRH, and VALRH. We then conducted an efficacy evaluation and survival analysis in an effort to investigate the feasibility of using minimally invasive techniques for patients with cervical cancer. Herein, we provide an evaluation of this surgical method and evaluate its efficacy for the surgical treatment of patients with early-stage cervical cancer. We present the following article in accordance with the STROBE reporting checklist (available at https://atm. amegroups.com/article/view/10.21037/atm-21-6450/rc). 


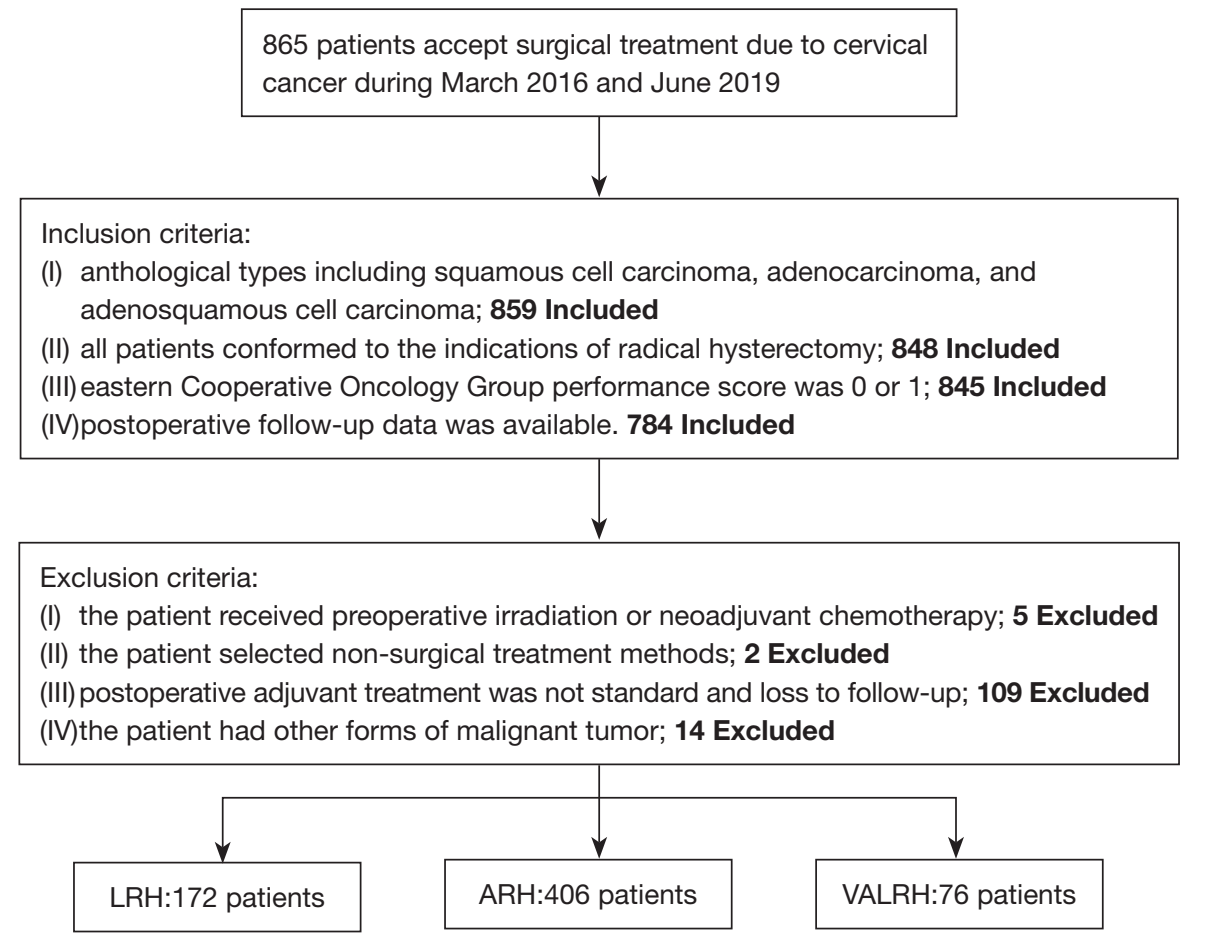

Figure 1 Study flow chart. LRH, laparoscopic radical hysterectomy; ARH, abdominal radical hysterectomy; VALRH, vaginal-assisted laparoscopic radical hysterectomy.

\section{Methods}

\section{Study design}

We retrospectively collected data from patients with cervical cancer who were admitted to the Liaoning Cancer Hospital \& China Medical University Cancer Hospital (Shengyang, China) between March 2016 and June 2019. Inclusion criteria: (I) anthological types including squamous cell carcinoma, adenocarcinoma, and adenosquamous cell carcinoma; (II) all patients conformed to the indications of radical hysterectomy; (III) Eastern Cooperative Oncology Group (ECOG) performance score of 0 or 1; and (IV) postoperative follow-up data were available. Also, we excluded 130 patients based on the following exclusion criteria: (I) patients who received preoperative irradiation or neoadjuvant chemotherapy; (II) patients who selected nonsurgical treatment methods; (III) cases where postoperative adjuvant treatment was not standard and loss to follow-up; or (IV) patients with other forms of malignant tumor. All surgeons in our team are skilled in the surgical methods carried out.

According to the surgical methods deployed, the patients were divided into three groups: VALRH, LRH, and ARH.
Of these, 406 patients underwent ARH, 172 underwent LRH, and 76 underwent VALRH (Figure 1). Classic surgery was performed in LRH and ARH. The surgical methods of VALRH were performed as follows: (I) Laparoscopy surgery: the entire operation was carried out using a laparoscope. We used a cup-shaped uterine manipulator instead of a uterine manipulator, as far as possible, to reduce squeezing of the tumor. Lymphadenectomy was performed by laparoscopy. Specifically, a long arm and high magnification were used to perform complete ligamentectomy for radical hysterectomy until vaginal disjunction. (II) Transvaginal surgery: tissue forceps were used to mark the vaginal wall to indicate a sufficient length for vaginal resection (see Figure 2). Hemostatic water (1:100,000 epinephrine solution) was injected into the full layer of the vaginal mucosa. Next, the full layer of the vaginal mucosa was cut open, and by using interrupted sutures, the vaginal wall was closed like a sleeve, and the neoplastic foci were also closed (Figure 3). Sharp and blunt dissection was performed ventrally, and the residual sacral ligament and visico-cervical ligament were removed, and complete radical hysterectomy was subsequently performed (Figure 4). The vagina and peritoneum were sutured with full-thickness annular sutures 
Page 4 of 14

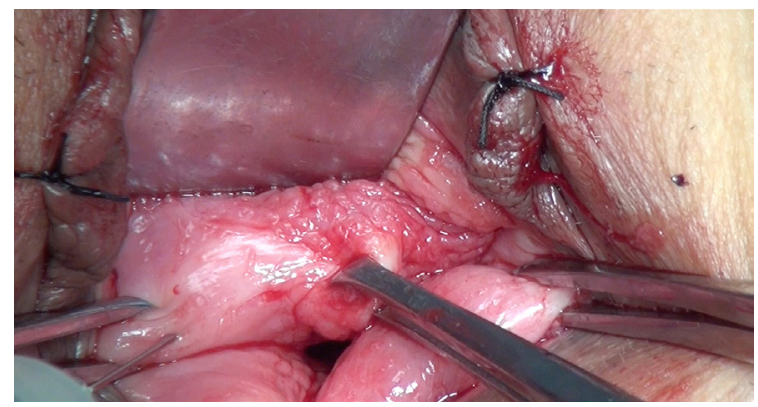

Figure 2 The vaginal wall was marked by tissue forceps.

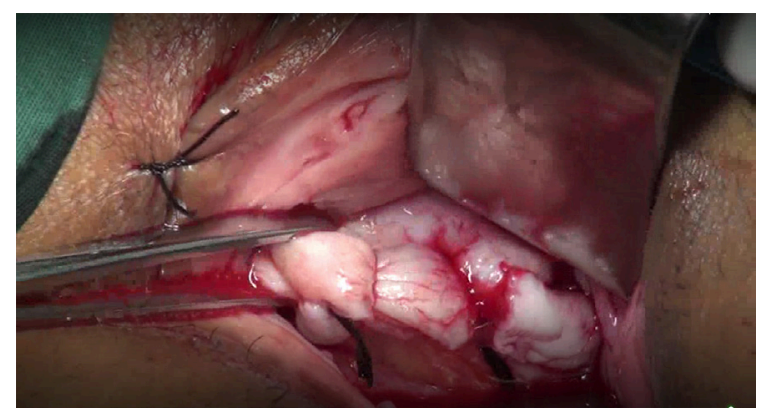

Figure 3 The vaginal wall was closed like a sleeve.

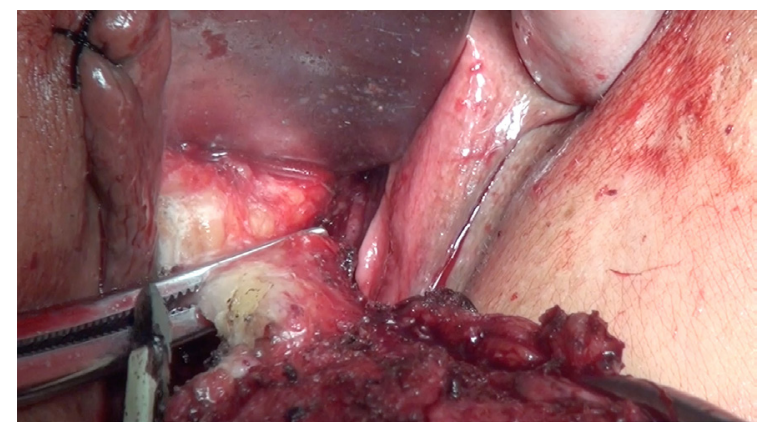

Figure 4 The residual sacral ligament and visico-cervical ligament were removed.

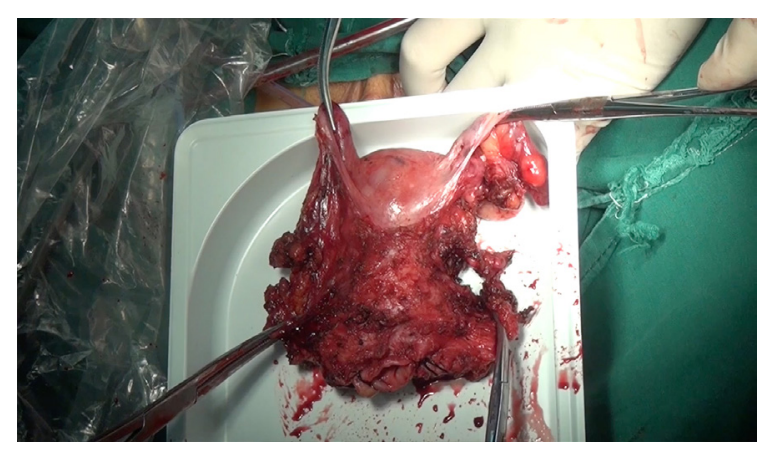

Figure 5 The specimens were removed surgically.
Wang et al. Efficacy evaluation of VALRH and LRH for cervical cancer

through the vagina; the sutures were then combined at the 12 and 6 clock points to ensure smooth pelvic drainage, and the operation was completed (Figure 5).

\section{Data collection}

All clinical datasets were complete, including age, stage, tumor size, histologic subtype, differentiation, infiltration, lymph vascular space invasion (LVSI), vaginal resection, lymph node metastasis, and adjuvant therapy, and the pathological diagnosis was confirmed. The final follow-up date was the 31st January 2021, and the median follow-up was 38 months. Medical information reviews, hospital follow-up, and telephone followup were conducted at the same timepoint to ensure that the survival outcome at follow-up was accurate. After surgery, all patients were followed-up every 3 months for 2 years, and then every 6 months thereafter. Follow-up evaluations included survival status, recurrence time, recurrence site (local pelvic recurrence or distant recurrence), time of death (if applicable), and cause of death (tumor-related deaths or non-tumor deaths, if applicable).

\section{Statistical analysis}

Patients undergoing ARH were used as the control group, while LRH or VALRH were used as the experimental groups. Data analysis was carried out with IBM SPSS Statistics version 26 (Chicago) Continuous variables were analyzed by Analysis of Variance (ANOVA). The Chisquared test or Fisher's $Z$ correction exact test were used as appropriate to compare categorical variables. Overall survival (OS) and disease-free survival (DFS) were calculated by the Kaplan-Meier method and compared using the logrank test. Multivariate analysis was performed using the Cox proportional hazards model. The results are presented as hazard ratios (HRs) with 95\% confidence intervals (95\% CIs). $\mathrm{P}$ values $<0.05$ were considered statistically significant. To exclude the decisive influence of individual surgeons on the overall outcome, we also performed sensitivity analysis. During this process, we eliminated patients from each surgeon one at a time, and then analyzed the differences between the three surgical styles. All tests were two-sided, and the results were considered statistically significant if $\mathrm{P}<0.05$.

\section{Ethical statement}

All procedures performed in this study involving human 
participants were in accordance with the Declaration of Helsinki (as revised in 2013). The study was approved by ethics board of Cancer Hospital of China Medical University (No. 2020G0322). Individual consent for this retrospective analysis was waived.

\section{Results}

In total, 865 individuals with cervical cancer underwent surgical treatment during that period. We included 784 patients according to the criteria. Our analysis showed that compared with the ARH group, patients in the LRH group were younger, diagnosed with an earlier clinical stage, had smaller lesions, and had lower requirements for supplementary adjuvant therapy after surgery (all $\mathrm{P}<0.05$ ). Furthermore, compared with the ARH group, patients in the VALRH group were younger, were diagnosed with an earlier clinical stage, had a greater extent of squamous cell carcinoma of the pathological type, had a longer length of vaginal resection, and had less need for adjuvant therapy after surgery (all $\mathrm{P}<0.05$ ). For LRH vs. ARH and VALRH $v s$. ARH, we found that there were no significant differences in body mass index (BMI), degree of differentiation, depth of infiltration, positive rate of LVSI, positive rate of vaginal margin, or positive rate of lymph node metastasis (all $\mathrm{P}>0.05$ ) (Table 1).

\section{Survival outcomes}

\section{Overall analysis}

The median follow-up was 38 months. Among the 654 patients, 27 cases died (4.2\%), including 20 cases in the ARH group (20/406, 4.9\%), four cases in the LRH group $(4 / 172,2.3 \%)$, and three cases in the VALRH group $(3 / 76,3.9 \%)$. There was no significant difference in the OS rate (all $\mathrm{P}>0.05, \mathrm{HR}=1.538,95 \%$ CI: $0.899-2.633$, $\mathrm{P}_{\text {LRH vs. ARH }}=0.144 ; \mathrm{HR}=0.771,95 \%$ CI: $0.229-2.569$, $\left.\mathrm{P}_{\text {VALRH vs. ARH }}=0.676\right)($ Table 2, Figure $6 A)$. A total of 55 patients $(55 / 654,8.4 \%)$ had a recurrence, including 27 patients $(27 / 40,6.7 \%)$ in the ARH group, 24 patients $(24 / 172,14.0 \%)$ in the LRH group, and four patients (4/76, $5.3 \%)$ in the VALRH group. The DFS rate in the ARH group was significantly higher than that in the LRH group ( $\mathrm{HR}=2.8$, 95\% CI: 1.199-3.607, $\mathrm{P}=0.004$ ); however, there was no significant difference between the VALRH and ARH groups ( $\mathrm{HR}=1.321,95 \% \mathrm{CI}: 0.462-3.777, \mathrm{P}=0.880$ ) (Table 2, Figure 6B).

Of the 55 patients who had a recurrence, 28 patients had distant recurrence and 27 patients had pelvic recurrence. In the ARH group, 21 patients had distant recurrence and 10 patients had pelvic recurrence. In the LRH group, four patients had distant recurrence and 20 patients had pelvic recurrence. In the VALRH group, three patients had distant recurrence and one patient had pelvic recurrence. There was a significant difference in the pelvic recurrence rate of LRH vs. ARH (HR =11.894, 95\% CI: 4.060-34.843, $\mathrm{P}<0.001)$, and no significant difference in the distant recurrence rate between these two groups $(\mathrm{HR}=1.226$, 95\% CI: $0.137-10.985, \mathrm{P}=0.078$ ). There was no significant difference in distant and pelvic recurrence rates of VALRH vs. ARH (HR $=0.402,95 \%$ CI: 0.139-1.162, $\mathrm{P}=0.855$; HR $=0.672,95 \%$ CI: $0.202-2.238, \mathrm{P}=0.514)$ (Table 2, Figure $6 C, 6 D)$.

\section{Subgroup analysis}

There were no significant differences in overall, pelvic, and distant recurrence rates for LRH vs. ARH and for VALRH vs. ARH patients in subgroups with a tumor size $<2 \mathrm{~cm}$ (all $\mathrm{P}>0.05)$. Compared with ARH, LRH had a higher overall recurrence rate $(\mathrm{P}<0.05)$ in the tumor sizes $\geq 2,<4$, lymph nodes negative, and without adjuvant therapy groups. Also, LRH had a higher local pelvic recurrence rate $(\mathrm{P}<0.05)$ in the tumor sizes $\geq 2,<4$, and $\geq 4 \mathrm{~cm}$ groups, irrespective of whether the lymph nodes were positive or not and whether adjuvant therapy was used. There was no difference in the distant recurrence rate of LRH compared to ARH among any of the subgroups $(\mathrm{P}>0.05)$. Furthermore, there were no significant differences in mortality, overall recurrence rate, local recurrence rate, and distant recurrence rate between the VALRH and ARH groups $(\mathrm{P}>0.05)$ (Table 3, Figure 7).

\section{Cox proportional-hazards models analysis}

Next, we used Cox proportional-hazards models to compare the survival outcomes between the three groups adjusted for age, BMI, FIGO stage, tumor size, LVSI, margins, lymph node metastases, and adjuvant therapy (Table 4). LRH surgery was identified as a high-risk factor for postoperative recurrence (HR $=2.338,95 \% \mathrm{CI}: 1.186-4.661, \mathrm{P}=0.014)$ and local pelvic recurrence (HR $=10.313,95 \%$ CI: 2.839-37.46, $\mathrm{P}<0.001$ ) (Table 4).

\section{Sensitivity analyses}

A total of nine surgeons operated on the 654 included patients. Sensitivity analysis was performed to examine 
Table 1 Baseline characteristics of the patients

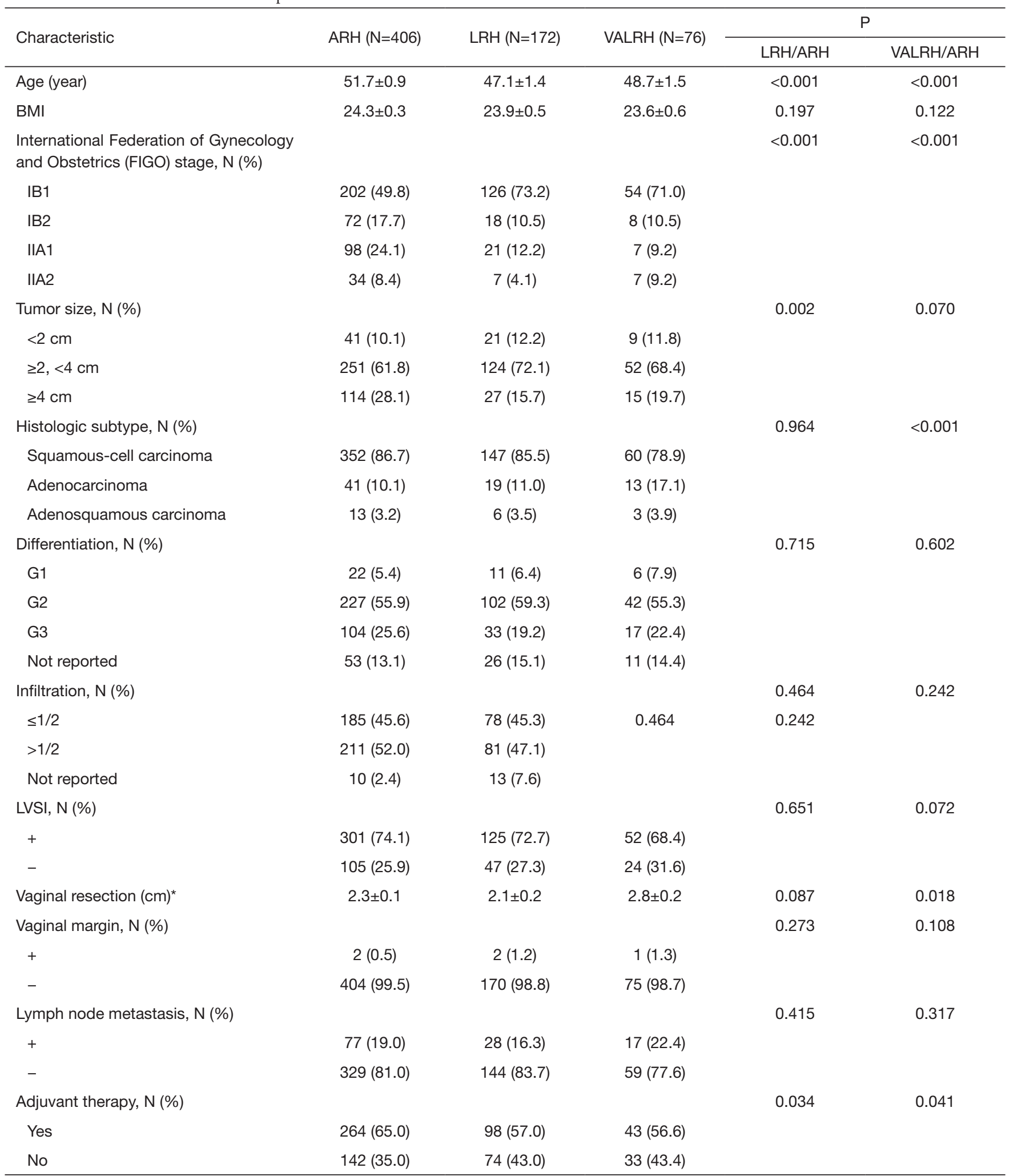

*, postoperative pathological measurement. $\mathrm{LRH}$, laparoscopic radical hysterectomy; ARH, abdominal radical hysterectomy; VALRH, vaginal-assisted laparoscopic radical hysterectomy; BMI, body mass index; LVSI, lymph vascular space invasion. 
Table 2 Overall survival and recurrence in each group (N, \%)

\begin{tabular}{lccc}
\hline Characteristic & ARH & LRH & VALRH \\
\hline Mortality rate & $4.9(20 / 406)$ & $2.3(4 / 172)$ & $3.9(3 / 76)$ \\
Overall recurrence rate & $6.7(27 / 406)$ & $14.0(24 / 172)$ & $5.3(4 / 76)$ \\
Distant recurrence rate & $5.2(21 / 406)$ & $2.3(4 / 172)$ & $3.9(3 / 76)$ \\
Pelvic recurrence rate & $1.5(6 / 406)$ & $11.6(20 / 172)$ & $1.3(1 / 76)$ \\
\hline
\end{tabular}

$\mathrm{LRH}$, laparoscopic radical hysterectomy; $\mathrm{ARH}$, abdominal radical hysterectomy; VALRH, vaginal-assisted laparoscopic radical hysterectomy.
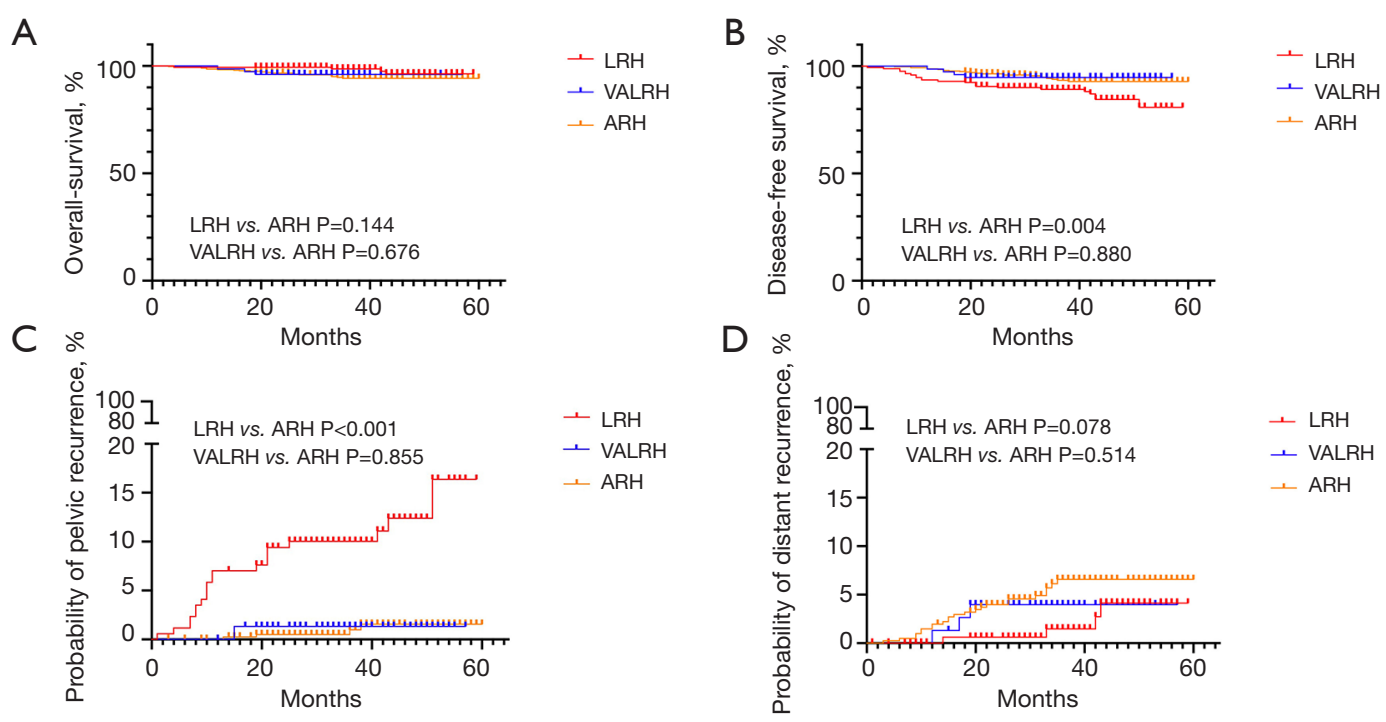

Figure 6 Overall estimates of OS and DFS. (A) OS; (B) DFS; (C) pelvic recurrence; (D) distant recurrence. OS, overall survival; DFS, disease-free survival. LRH, laparoscopic radical hysterectomy; ARH, abdominal radical hysterectomy; VALRH, vaginal-assisted laparoscopic radical hysterectomy.

the robustness of our results. During this process, we eliminated patients from each surgeon one at a time and analyzed the differences between the three surgical styles. The outcome measures were local pelvic recurrence (PR), distant recurrence (DR), and overall recurrence. Our conclusions did not change following this analysis. LRH had a higher pelvic recurrence rate and a poorer DFS than $\mathrm{ARH}$, but there were no differences between VALRH and ARH. These differences in survival outcomes may be due to the surgical approach itself (Figure 8).

\section{Discussion}

With the popularization of minimally invasive techniques, minimally invasive surgery for cervical cancer has gradually become a commonly applied approach. Numerous previous studies have reported similar outcomes between LRH and open surgery $(13,14)$. However, two studies published in the New England Fournal of Medicine in 2018 represented a significant turning point. One prospective and multicenter randomized clinical trial (RCT), reported by Ramirez et al. (4) and carried out at the Anderson Research Center, showed that a minimally invasive surgery group had a higher local recurrence rate and a lower DFS and OS than open surgery. Subsequently, Melamed et al. (5) from Harvard University published another large data study, the Real World Study (RWS) using data derived from the National Cancer Database and National Cancer Institute of the United States. Their results suggested that with the development of laparoscopic cervical cancer surgery in 2006, the overall survival rate of cervical cancer exhibited a downward trend. However, these two studies did not explain 


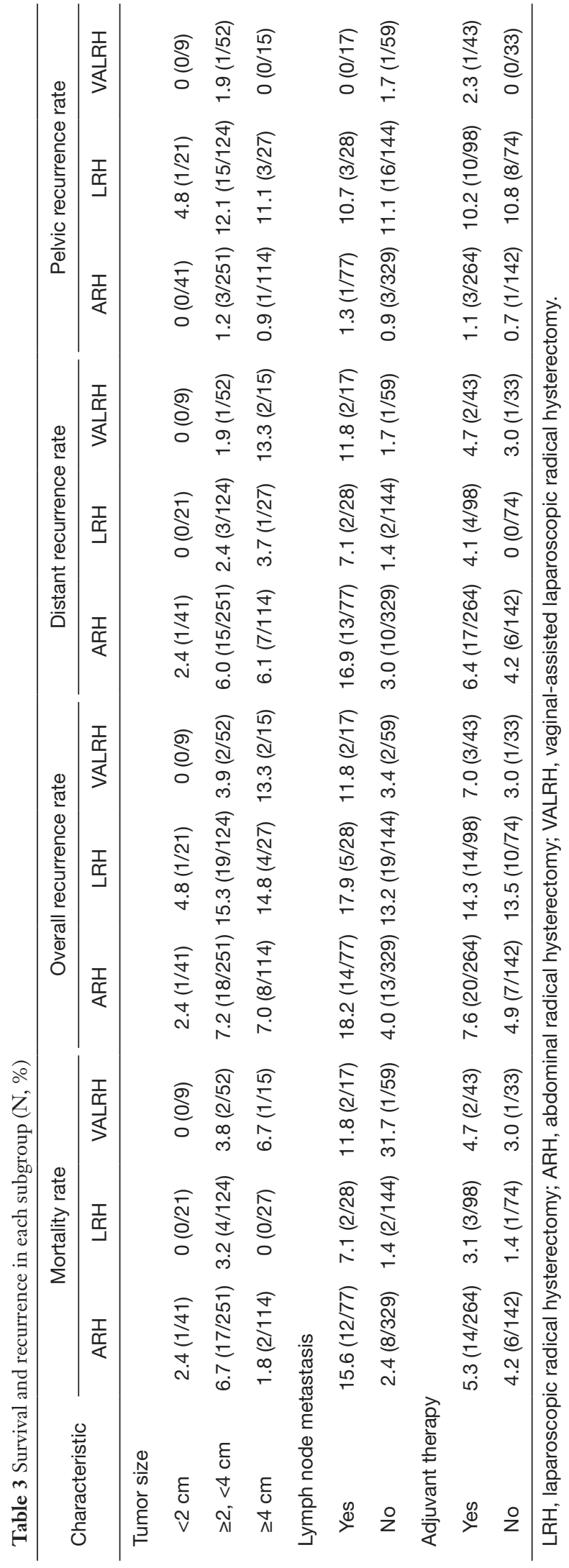

why minimally invasive surgery was associated with shorter survival outcomes, and there were also some controversies and limitations. In addition, cervical cancer patients recover faster and have less pain after LRH surgery, indicating that many cancer patients can avoid the problems associated with huge surgical trauma. The comprehensive harm caused by open surgery, such as the huge incision and longterm exposure of the abdominal organs, as well as internal syndrome, infection syndrome, and blood transfusion risk, is far greater than that caused by laparoscopy (15). Therefore, we should not completely deny the benefits of minimally invasive surgery for patients, but rather attempt to identify the possible causes for the high local recurrence rate, low DFS, and low OS after minimally invasive surgery, and how to avoid these issues.

Some studies have reported that after avoiding the exposure of tumors in the pelvic cavity, the prognosis of laparoscopic surgery can be consistent with that of open surgery (16). By reviewing the procedure used for LRH surgery, we believe that the tumor exposure caused by vaginal amputation in the open state of laparoscopic surgery may violate the requirement of the tumor-free concept in malignant tumor surgery, thus resulting in an increased local recurrence rate for laparoscopic surgery, thereby leading to a low OS. VALRH, as a combination of laparoscopic and vaginal minimally invasive techniques, avoids tumor exposure by transvaginal closure of the lesion, which can make up for the exposure of the tumor by laparoscopically open vaginal dissection. The long arm amplification provided by laparoscopic surgery also makes up for the technical difficulties caused by the poor field of vision and retro-level anatomy in vaginal surgery. VALRH cannot only meet the principle of no tumor but also involve the technical advantages of laparoscopy. Studies have suggested that the use of a uterine manipulator can promote the micrometastasis of tumor cells $(8,9)$. However, most cervical cancer patients with a normal uterine size and good mobility require dissection surgery, and a uterine manipulator is not necessary. Intraoperative suspension and the auxiliary pull arm technology provided by VALRH can completely replace the uterine manipulator (7). Studies have also suggested that laparoscopic surgery may further promote the implantation and metastasis of tumor cells due to the presence of $\mathrm{CO}_{2}$ circulating in the pneumoperitoneum $(10,11)$. However, Lin et al. stimulated cervical cancer cells in vitro in a $\mathrm{CO}_{2}$ pneumoperitoneum environment and observed significantly increased proliferation ability after a short period of proliferation inhibition. However, there was a reduction 
A

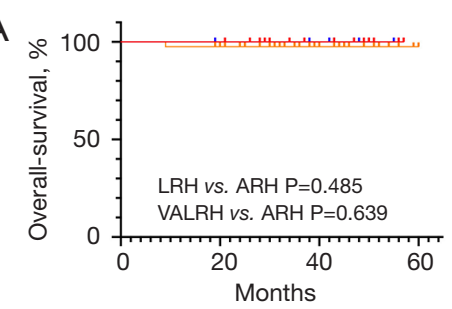

- LRH

- VALRH

$+\mathrm{ARH}$

B

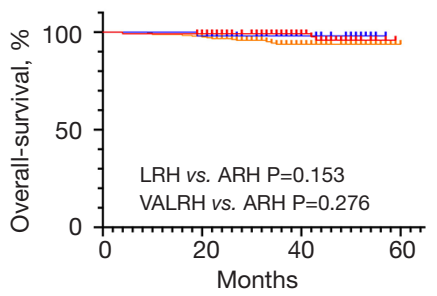

- LRH

$\rightarrow$ VALRH

$+\mathrm{ARH}$

C

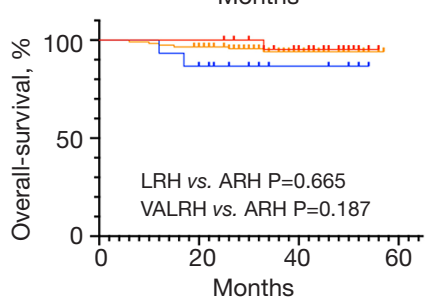

D

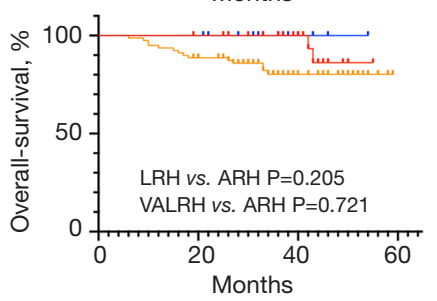

E

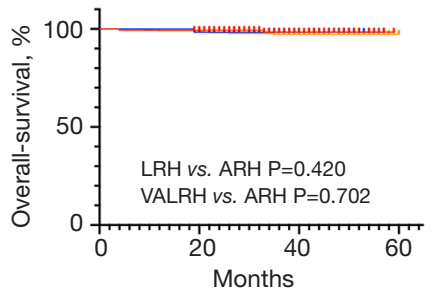

F

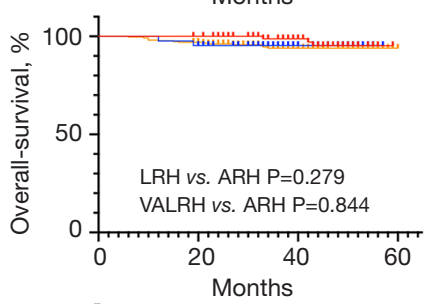

$+\mathrm{LRH}$

- VALRH

$\perp$ ARH

$+\mathrm{LRH}$

$\perp$ VALRH

- ARH

$+\mathrm{LRH}$

- VALRH

- $\mathrm{ARH}$

(2)

$+\mathrm{LRH}$

- VALRH

- ARH

$$
\text { ARH }
$$
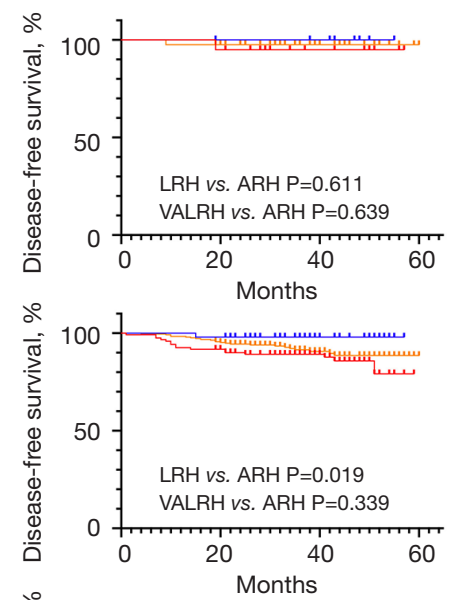

$+\mathrm{LRH}$

- VALRH

- ARH

- LRH

- VALRH

- ARH

ARH
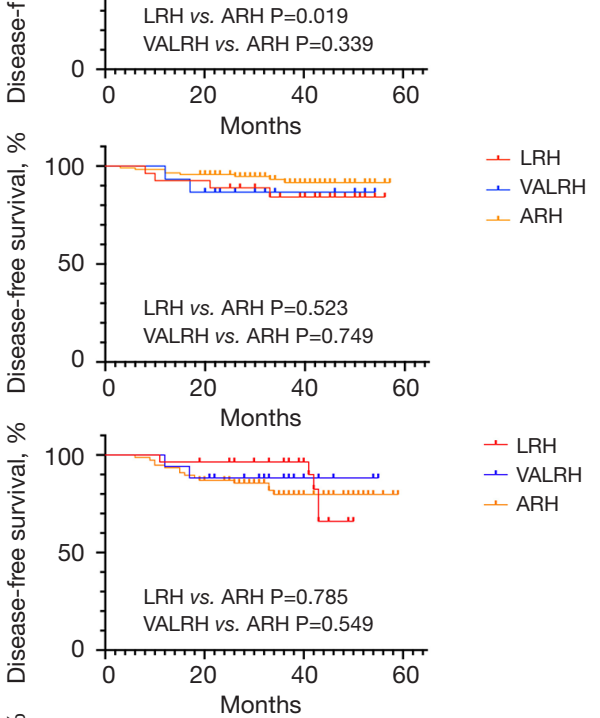

$+\mathrm{LRH}$

$\sim$ VALRH

$+\mathrm{ARH}$

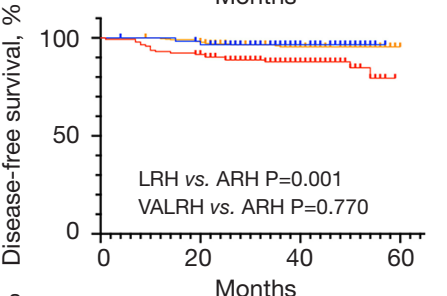

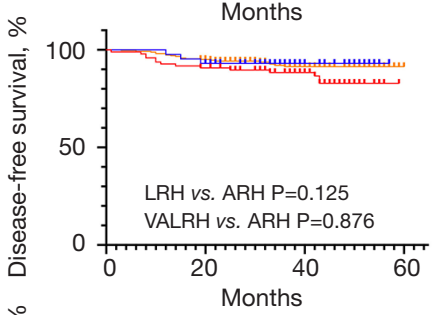

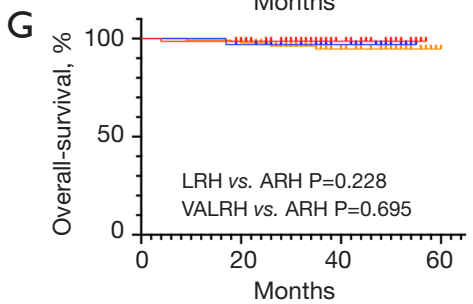

$+\mathrm{LRH}$

- VALRH

- ARH

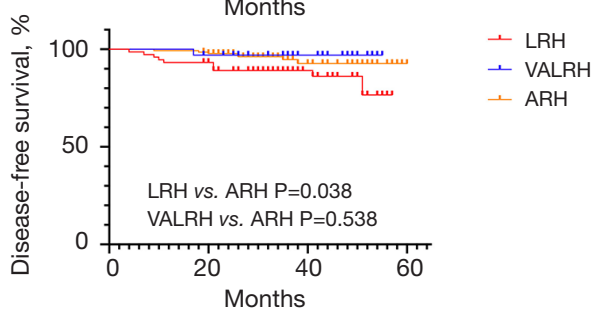

Figure 7 Estimates of OS and DFS for different subgroups. (A) Tumor size $<2 \mathrm{~cm}$; (B) tumor sizes $\geq 2,<4 \mathrm{~cm}$; (C) tumor sizes $\geq 4 \mathrm{~cm}$; (D) positive lymph nodes; (E) negative lymph nodes; (F) adjuvant therapy; (G) no adjuvant therapy. LRH, laparoscopic radical hysterectomy; ARH, abdominal radical hysterectomy; VALRH, vaginal-assisted laparoscopic radical hysterectomy; OS, overall survival; DFS, disease-free survival. 
Table 4 Cox proportional-hazards models analysis

\begin{tabular}{|c|c|c|c|c|c|c|}
\hline Characteristic & \multicolumn{3}{|c|}{ LRH vs. ARH } & \multicolumn{3}{|c|}{ VALRH vs. ARH } \\
\hline Overall recurrence & 2.338 & $1.186-4.661$ & 0.014 & 0.791 & $0.263-2.380$ & 0.676 \\
\hline Distant recurrence & 0.709 & $0.230-2.182$ & 0.549 & 0.634 & $0.173-2.321$ & 0.492 \\
\hline Pelvic recurrence & 10.313 & $2.839-37.46$ & $<0.001$ & 1.343 & $0.136-13.232$ & 0.800 \\
\hline
\end{tabular}

$\mathrm{LRH}$, laparoscopic radical hysterectomy; $\mathrm{ARH}$, abdominal radical hysterectomy; VALRH, vaginal-assisted laparoscopic radical hysterectomy; HR, hazard ratio; $95 \% \mathrm{Cl}, 95 \%$ confidence interval.

LRH vs. ARH (PR)

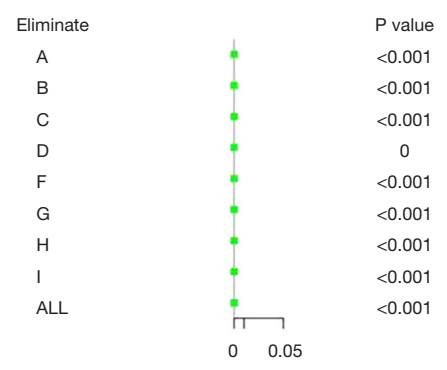

LRH vs. ARH (DR)

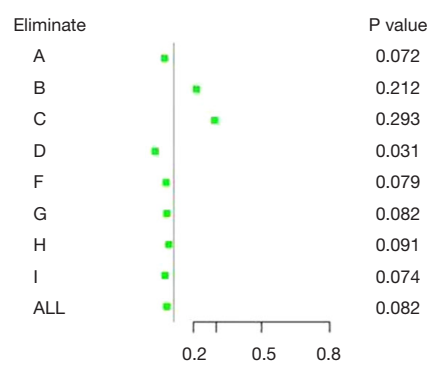

LRH vs. ARH (Recurrence)

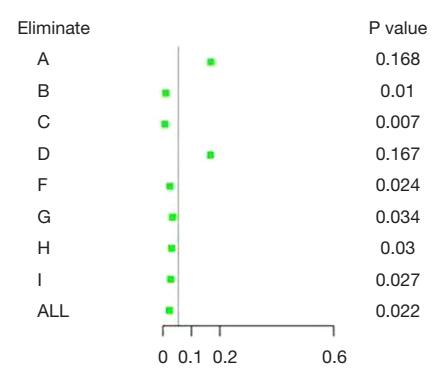

VALRH vs. ARH (PR)

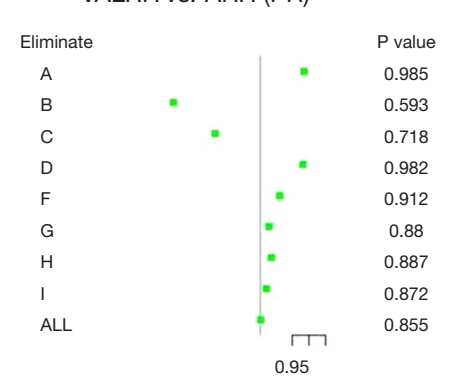

VALRH vs. ARH (DR)

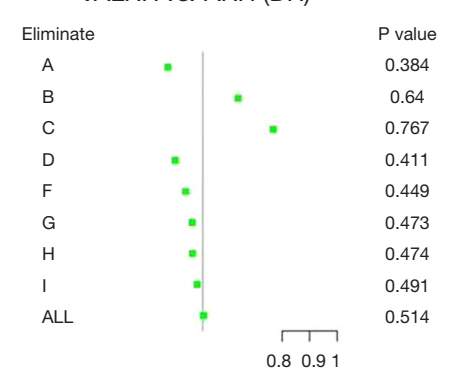

VALRH vs. ARH (Recurrence)

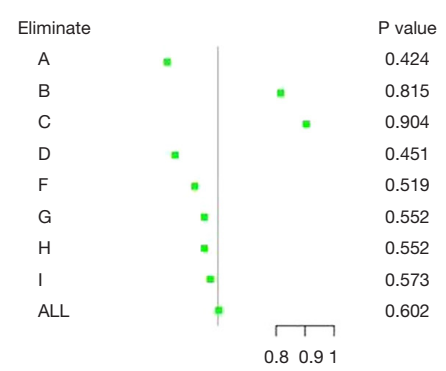

Figure 8 Sensitivity analysis for each surgeon. Each marker point represents the $\mathrm{P}$ value reanalyzed following the exclusion of patients from each surgeon one at a time. The results showed that no single surgeon had a significant impact on the final study conclusion. During this process, we eliminated patients from each surgeon one at a time and analyzed the differences between the three surgical styles. Considering that all VALRH procedures were performed by surgeon E, and all patients of surgeon E underwent VALRH, we would be unable to compare VALRH with the other surgical styles if surgeon E was excluded. Therefore, surgeon E was not included in the sensitivity analysis. LRH, laparoscopic radical hysterectomy; ARH, abdominal radical hysterectomy; VALRH, vaginal-assisted laparoscopic radical hysterectomy; PR, pelvic recurrence; DR, distant recurrence. 
in their invasion, migration, and adhesion ability, although there were no significant correlations with $\mathrm{CO}_{2}$ pressure (17). Studies of endometrial cancer have confirmed that $\mathrm{CO}_{2}$ circulating in the pneumoperitoneum does not increase the risk of tumor recurrence and death (18), and therefore provides a certain basis for the application of laparoscopic surgery in cervical cancer. Based on these studies, we believe that VALRH may be used for radical surgery in cervical cancer patients by meeting the requirements of nontumor exposure and minimally invasive operation, and thus reduce the technical difficulty of surgery without affecting the prognosis of patients and avoiding significant surgical trauma.

To further verify this speculation, we selected patients who received LRH, VALRH, and ARH in our hospital between March 2016 and June 2019. The surgeons were all experienced chief physicians in oncology hospitals. The overall operation format and postoperative adjuvant therapy were standardized and balanced, the patient followup information was complete, and the overall mortality was $4.2 \%$. The results were consistent with that of LACC, which indicated that the patients selected in this paper were satisfied with the combined treatment of cervical cancer based on surgery. In this study, the mortality rate of ARH was higher than that of LRH and VALRH, but the difference was not statistically significant. The main reason is that the ARH group had later FIGO stage, larger cervical lesions, higher incidence of LVSI and lymph node metastasis, leading to lower overall survival rate of the ARH group. Compared with ARH, LRH had a higher pelvic local recurrence rate, although there was no significant difference in distant recurrence between the two groups. However, due to a significant increase in pelvic recurrence rate, the DFS of LRH was shorter than ARH, which was consistent with the findings of the LACC experiments (4). In this study, the prognosis of patients in the VALRH surgery group was comparable with that of those in the ARH group, which confirmed our hypothesis. There were no significant differences in the overall recurrence rate, local recurrence rate, distant recurrence rate, or survival rate between the VALRH and ARH groups, thus indicating that VALRH could precisely avoid the disadvantages of total laparoscopic surgery. VALRH directly targets lesions and creates a closed tumor vaginal cuff, thereby ensuring the safety of a $3 \mathrm{~cm}$ resection range, which avoids tumor exposure caused by vaginal opening in laparoscopic operations. Furthermore, VALRH does not require a spiral uterine manipulator and reduces tumor contact and extrusion, thus reducing the risk of tumor micrometastases (19-21).

A previous retrospective study reported similar results for laparoscopic-assisted vaginal extensive hysterectomy. The surgery was performed within the vagina to cuff- package lesions and avoided the extrusion of tumors by a spiral uterine manipulator, thus achieving the same oncological prognosis as open surgery $(22,23)$; our data concurred with these previous results. However, laparoscopic-assisted vaginal extensive hysterectomy requires transvaginal removal of the uterine ligament and free the ureteral. This makes the operation more difficult as there are high technical requirements, which means that there is a long learning curve for the surgeon and more intraoperative complications, thus limiting the clinical application of this technique. In contrast, when using VALRH, we only need to cut the vaginal edge and there is less transvaginal operation. Moreover, this surgery is not technically difficult and is therefore more suitable for clinical promotion. We also conducted sensitivity analysis for different operators and reached a consistent conclusion, even after excluding the influence of the surgical experience of different operators, which further confirmed the stability of our data. This provides us with a confident basis from which to carry out prospective clinical trials to validate our current findings.

In view of the lack of stratified prognostic data for both the LACC and RWS studies, as well as the comprehensive, authentic, and reliable follow-up data provided by this study, we also conducted subgroup analysis and found that when the lesion was $<2 \mathrm{~cm}$, there was no statistical significance in the overall recurrence rate, distant recurrence rate, and local pelvic recurrence rate between LRH $v s$. ARH and VALRH $v s$. ARH. Limited by the fact that, as a tumor specialized hospital, there were relatively few earlier cases and fewer recurrent patients, this study could not confirm the differences in the prognosis of tumor patients with a lesion $<2 \mathrm{~cm}$ based on the three surgical methods. Of course, this does not exclude the fact that patients at an earlier stage are suitable for routine total laparoscopic surgery. When lesions were $\geq 2,<4 \mathrm{~cm}$, postoperative lymph nodes were negative, and there was no postoperative auxiliary treatment group, the overall recurrence and local recurrence rates of LRH were higher than ARH. When lesions were $\geq 4 \mathrm{~cm}$, postoperative lymph nodes were positive, and auxiliary treatment was provided postoperatively, LRH also failed to avoid a higher local recurrence rate in the pelvis compared to ARH. There was also no difference in the distant recurrence rate, further 
confirming that local tumor exposure or compression may promote local recurrence. These findings were consistent with previous reported research (24-27). However, there was no difference in the outcomes between VALRH and $\mathrm{ARH}$, further demonstrating that improved techniques may mean that minimally invasive surgery could be applied more to patients with cervical cancer. However, more prospective clinical trials are needed to further verify the efficacy of laparoscopic surgery in patients with cervical cancer, especially for "low-risk" patients, so as to improve the indications for minimally invasive surgery and the strict control of operative methods for early cervical cancer. This could allow more patients with cervical cancer to benefit from minimally invasive surgery.

There are still some limitations to this study that need to be considered. Firstly, our data was obtained from a single-center and our study was retrospective. Therefore, more prospective and multi-center studies are needed to further verify the efficacy of VALRH for the surgical treatment of cervical cancer. Also, the median follow-up interval in our study was short; therefore, longer followup analysis is still needed. Due to the number of cases, the safety of laparoscopic surgery for "low-risk" patients with early cervical cancer could not be fully demonstrated in the subgroup with a tumor diameter $<2 \mathrm{~cm}$. Further studies and additional data are now needed for confirmation.

Laparoscopic surgery for cervical cancer faces huge challenges and some scholars have highlighted suggestions for improving the concept of disease-free surgical options (28). VALRH cannot only meet the principle of no tumor but also involve the technical advantages of laparoscopy, moreover, its minimally invasive advantage is not possessed by ARH, but it requires the surgeon master certain vaginal operation skills. In view of the poor prognosis suffered by LRH, the great surgical trauma challenge of ARH and the minimally invasive and tumorfree advantages of VALRH, we compared the effects of three surgical methods on recurrence and survival of cervical cancer, found that VALRH had the same prognosis with ARH and better than LRH, our findings provide proof for this concept and suggest that VALRH can solve the problems created by total laparoscopic surgery and tumor exposure by improving survival outcomes and reducing the local recurrence rate. Therefore, for patients with early cervical cancer, regardless of the size of cervical lesions, VALRH surgery can be selected. The prognosis is similar to $\mathrm{ARH}$ and surgical trauma can be reduced, which is worthy of clinical application.

\section{Acknowledgments}

Funding: This work was supported by a grant from the Key R \& D projects of Liaoning Province (2018225037).

\section{Footnote}

Provenance and Peer Review: This article was commissioned by the editorial office, Annals of Translational Medicine for the series "New Progress and Challenge in Gynecological Cancer". The article has undergone external peer review.

Reporting Checklist: The authors have completed the STROBE reporting checklist. Available at https://atm. amegroups.com/article/view/10.21037/atm-21-6450/rc

Data Sharing Statement: Available at https://atm.amegroups. com/article/view/10.21037/atm-21-6450/dss

Conflicts of Interest: All authors have completed the ICMJE uniform disclosure form (available at https://atm. amegroups.com/article/view/10.21037/atm-21-6450/coif). The series "New Progress and Challenge in Gynecological Cancer" was commissioned by the editorial office without any funding or sponsorship. DW served as the unpaid Guest Editor of the series. The authors have no other conflicts of interest to declare.

Ethical Statement: The authors are accountable for all aspects of the work in ensuring that questions related to the accuracy or integrity of any part of the work are appropriately investigated and resolved. All procedures performed in this study involving human participants were in accordance with the Declaration of Helsinki (as revised in 2013). The study was approved by ethics board of Cancer Hospital of China Medical University (No. 2020G0322). Individual consent for this retrospective analysis was waived.

Open Access Statement: This is an Open Access article distributed in accordance with the Creative Commons Attribution-NonCommercial-NoDerivs 4.0 International License (CC BY-NC-ND 4.0), which permits the noncommercial replication and distribution of the article with the strict proviso that no changes or edits are made and the original work is properly cited (including links to both the formal publication through the relevant DOI and the license). See: https://creativecommons.org/licenses/by-nc-nd/4.0/. 


\section{References}

1. Abu-Rustum NR, Yashar CM, Bean S, et al. NCCN Guidelines Insights: Cervical Cancer, Version 1.2020. J Natl Compr Canc Netw 2020;18:660-6.

2. Conrad LB, Ramirez PT, Burke W, et al. Role of Minimally Invasive Surgery in Gynecologic Oncology: An Updated Survey of Members of the Society of Gynecologic Oncology. Int J Gynecol Cancer 2015;25:1121-7.

3. Diver E, Hinchcliff E, Gockley A, et al. Minimally Invasive Radical Hysterectomy for Cervical Cancer Is Associated With Reduced Morbidity and Similar Survival Outcomes Compared With Laparotomy. J Minim Invasive Gynecol 2017;24:402-6.

4. Ramirez PT, Frumovitz M, Pareja R, et al. Minimally Invasive versus Abdominal Radical Hysterectomy for Cervical Cancer. N Engl J Med 2018;379:1895-904.

5. Melamed A, Margul DJ, Chen L, et al. Survival after Minimally Invasive Radical Hysterectomy for Early-Stage Cervical Cancer. N Engl J Med 2018;379:1905-14.

6. Wenzel HHB, Smolders RGV, Beltman JJ, et al. Survival of patients with early-stage cervical cancer after abdominal or laparoscopic radical hysterectomy: a nationwide cohort study and literature review. Eur J Cancer 2020;133:14-21.

7. Baeten I, Hoogendam JP, Schreuder H, et al. The influence of learning curve of robot-assisted laparoscopy on oncological outcomes in early-stage cervical cancer: an observational cohort study. BJOG 2021;128:563-71.

8. Volz J, Köster S, Spacek Z, et al. The influence of pneumoperitoneum used in laparoscopic surgery on an intraabdominal tumor growth. Cancer 1999;86:770-4.

9. Kong TW, Chang SJ, Piao X, et al. Patterns of recurrence and survival after abdominal versus laparoscopic/robotic radical hysterectomy in patients with early cervical cancer. J Obstet Gynaecol Res 2016;42:77-86.

10. Bogani G, Ghezzi F, Chiva L, et al. Patterns of recurrence after laparoscopic versus open abdominal radical hysterectomy in patients with cervical cancer: a propensitymatched analysis. Int J Gynecol Cancer 2020;30:987-92.

11. Pedone Anchora L, Bizzarri N, Kucukmetin A, et al. Investigating the possible impact of peritoneal tumor exposure amongst women with early stage cervical cancer treated with minimally invasive approach. Eur J Surg Oncol 2021;47:1090-7.

12. Ramirez PT, Schmeler KM, Soliman PT, et al. Fertility preservation in patients with early cervical cancer: radical trachelectomy. Gynecol Oncol 2008;110:S25-8.

13. Arimoto T, Kawana K, Adachi K, et al. Minimization of curative surgery for treatment of early cervical cancer: a review. Jpn J Clin Oncol 2015;45:611-6.

14. Saltari M, Corrado G, Patrizi L, et al. Comment: Survival rate comparisons amongst cervical cancer patients treated with an open, robotic-assisted or laparoscopic radical hysterectomy: A five year experience. Surg Oncol 2016;25:244-5.

15. Uppal S, Rebecca Liu J, Kevin Reynolds R, et al. Trends and comparative effectiveness of inpatient radical hysterectomy for cervical cancer in the United States (2012-2015). Gynecol Oncol 2019;152:133-8.

16. Wang Y, Wang A, Zhan J, et al. Curative effect of laparoscopic-assisted vaginal radical trachelectomy combined with pelvic lymph node dissection on early-stage cervical cancer. J BUON 2021;26:684-90.

17. Lin F, Pan L, Li L, et al. Effects of a simulated CO2 pneumoperitoneum environment on the proliferation, apoptosis, and metastasis of cervical cancer cells in vitro. Med Sci Monit 2014;20:2497-503.

18. Fu C, Li GY, Liu FY, et al. Effect of carbon dioxide pneumoperitoneum-laparoscopic surgery on tumor seeding and metastases in endometrial cancer. Zhong Nan Da Xue Xue Bao Yi Xue Ban 2008;33:130-7.

19. Kanao H, Matsuo K, Aoki Y, et al. Feasibility and outcome of total laparoscopic radical hysterectomy with no-look notouch technique for FIGO IB1 cervical cancer. J Gynecol Oncol 2019;30:e71.

20. Kohler C, Hertel H, Herrmann J, et al. Laparoscopic radical hysterectomy with transvaginal closure of vaginal cuff - a multicenter analysis. Int J Gynecol Cancer 2019;29:845-50.

21. Gottschalk E, Lanowska M, Chiantera V, et al. Vaginalassisted laparoscopic radical hysterectomy: rationale, technique, results. JSLS 2011;15:451-9.

22. Hertel H, Köhler C, Michels W, et al. Laparoscopicassisted radical vaginal hysterectomy (LARVH): prospective evaluation of 200 patients with cervical cancer. Gynecol Oncol 2003;90:505-11.

23. Pahisa J, Martínez-Román S, Torné A, et al. Comparative study of laparoscopically assisted radical vaginal hysterectomy and open Wertheim-Meigs in patients with early-stage cervical cancer: eleven years of experience. Int J Gynecol Cancer 2010;20:173-8.

24. Steed H, Rosen B, Murphy J, et al. A comparison of laparascopic-assisted radical vaginal hysterectomy and radical abdominal hysterectomy in the treatment of cervical cancer. Gynecol Oncol 2004;93:588-93.

25. Choi CH, Lee JW, Lee YY, et al. Comparison of 
laparoscopic-assisted radical vaginal hysterectomy and laparoscopic radical hysterectomy in the treatment of cervical cancer. Ann Surg Oncol 2012;19:3839-48.

26. Kim SI, Cho JH, Seol A, et al. Comparison of survival outcomes between minimally invasive surgery and conventional open surgery for radical hysterectomy as primary treatment in patients with stage IB1-IIA2 cervical cancer. Gynecol Oncol 2019;153:3-12.

Cite this article as: Wang $\mathrm{H}$, Li D, Wang C, Wang $\mathrm{X}$, Yu M, Zhang X, Li L, Zeng Q, Long Z, Zheng W, Liu G, Wang D. Efficacy evaluation of vaginal-assisted laparoscopic radical hysterectomy and laparoscopic radical hysterectomy for cervical cancer: a single-center retrospective case series study. Ann Transl Med 2022;10(2):124. doi: 10.21037/atm-21-6450
27. Chen C, Liu P, Ni Y, et al. Laparoscopic versus abdominal radical hysterectomy for stage IB1 cervical cancer patients with tumor size $\leq 2 \mathrm{~cm}$ : a case-matched control study. Int J Clin Oncol 2020;25:937-47.

28. Bebia V, Monreal-Clua S, Pérez-Benavente A, et al. Potential strategies for prevention of tumor spillage in minimally invasive radical hysterectomy. J Gynecol Oncol 2020;31:e73. 